\title{
Evaluation of peptidylarginine deiminase 4 concentration and PADI4 polymorphisms in sepsis- induced acute kidney injury
}

Nara Aline Costa ( $\square$ na.costa@unesp.br)

Universidade Estadual Paulista Julio de Mesquita Filho https://orcid.org/0000-0001-8159-1209

Bertha Furlan Polegato

Universidade Estadual Paulista Julio de Mesquita Filho

Amanda Gomes Pereira

Universidade Estadual Paulista Julio de Mesquita Filho

Rodrigo Velloni da Silva Bastos

Universidade Estadual Paulista Julio de Mesquita Filho

Sérgio Alberto Rupp de Paiva

Universidade Estadual Paulista Julio de Mesquita Filho

Ana Lucia Gut

Universidade Estadual Paulista Julio de Mesquita Filho

André Luis Balbi

Universidade Estadual Paulista Julio de Mesquita Filho

\section{Daniela Ponce}

Universidade Estadual Paulista Julio de Mesquita Filho

Leonardo Antônio Mamede Zornoff

Universidade Estadual Paulista Julio de Mesquita Filho

Paula Schmidt Azevedo

Universidade Estadual Paulista Julio de Mesquita Filho

Marcos Ferreira Minicucci

Universidade Estadual Paulista Julio de Mesquita Filho

\section{Research article}

Keywords: acute kidney injury; peptidylarginine deiminase 4; sepsis; mortality; dialysis

Posted Date: November 7th, 2019

DOI: https://doi.org/10.21203/rs.2.16993/v1 
License: (c) (i) This work is licensed under a Creative Commons Attribution 4.0 International License. Read Full License 


\section{Abstract}

Background: The influence of PAD4 concentration and its polymorphisms in SAKI development are poorly evaluated. Thus, the aim of this study is to evaluate the PAD 4 concentration and PADI4 polymorphisms, as predictors of AKI development, need for renal replacement therapy (RRT), and mortality in patients with septic shock. Methods: We included all individuals aged $\geq 18$ years, with the diagnosis of septic shock at ICU admission. Blood samples were taken within the first 24 hours of the patient's admission to determine serum PAD4 concentration and its polymorphism PADI4 (rs11203367) and (rs874881). Patients were followed during their ICU stay and the development of SAKI was evaluated. Among the patients in whom SAKI developed, mortality and need for RRT were also evaluated. Results: 99 patients were included in the analysis. SAKI developed in approximately $51.5 \%$ of patients during the ICU stay; of these, $21.5 \%$ required RRT and $80 \%$ died. There was no difference between PAD4 concentration ( $p=$ $0.116)$ and its polymorphisms rs11203367 ( $p=0.910)$ and rs874881 ( $=0.769)$ in patients in whom SAKI did or did not develop. However, PAD4 had a positive correlation with plasma urea concentration $(r=$ 0.269 and $p=0.007)$ and creatinine $(r=0.284$ and $p=0.004)$. The PAD 4 concentration and PADI4 polymorphisms were also not associated with RRT and with mortality in patients with SAKI. Conclusion: PAD4 concentration and its polymorphisms were not associated with SAKI development, the need for RRT, or mortality in patients with septic shock. However, PAD4 concentrations were associated with creatinine and urea levels in these patients.

\section{Background}

Sepsis-induced acute kidney injury (SAKI) represents one of the most common complications of sepsis and is associated with a worse prognosis and with mortality rates ranging from $50 \%$ to $70 \%$ [1]. Despite intense research, there is currently no effective therapy or preventive measures for SAKI [2]. The physiopathology of SAKI is multifactorial and complex, involving changes in renal hemodynamics, endothelial dysfunction, infiltration of inflammatory cells into the renal parenchyma, intraglomerular thrombosis, and tubular cell necrosis [3]. Indeed, renal inflammation and injury are mediated by upregulation of proinflammatory cytokines followed by leukocyte infiltration, including T lymphocytes, natural killer cells, and neutrophils [4]. It should be reinforced that infiltrating neutrophils play a major role in renal tubular inflammation and cell death [4]. In fact, blocking or neutralizing neutrophils has been shown to attenuate the severity and duration of ischemic acute kidney injury $(A K I)[5,6]$.

Thus, the understanding of pathways that modulate the inflammatory process could improve the current knowledge in physiopathology SAKI and identify new therapeutic targets. In this scenario, the citrullination process deserves to be highlighted because it has been identified in several inflammatory diseases $[7,8]$. Citrullination is the posttranslational modification of peptidyl arginine to peptidyl citrulline, catalyzed by the family of enzymes peptidyl arginine deiminase (PAD) [9]. In humans and rodents, there are five PAD subtypes. Among them, PAD4 has been widely studied in innate immunity and autoimmune diseases as multiple sclerosis, lupus, ulcerative colitis and rheumatoid arthritis. [10] 
PAD4 was first identified in the nucleus of a neutrophil-like cell line (HL-60) [10], and PAD4 mediated citrullination of core histones $(\mathrm{H} 3, \mathrm{H} 4$, and $\mathrm{H} 2 \mathrm{~A}$ ) is critical for neutrophil extracellular traps (NETs) formation after bacterial infection [11]. In the kidney, PAD2 and PAD4 messenger RNAs are present, although only PAD4 protein expression has been detected [12,13]. Ham et al. [14], demonstrated that PAD4 plays an important role in a model of renal ischemia-reperfusion injury by increasing tubular inflammatory response. Based on previous data published by our group, using the same dataset, higher PAD4 concentrations were associated with lower ICU survival in patients with septic shock [15].

Despite PAD4 concentration, PADI4 polymorphisms could influence enzyme activity. In this context, some polymorphisms of PADI4 gene have been found in humans. The PADI4 gene is located on the short arm of chromosome 1 at position 36.13 and functional polymorphisms, such as rs11203367 and rs874881, have been associated with susceptibility to rheumatoid arthritis $[16,17]$.

However, to our knowledge, there are no clinical studies that have evaluated the influence of PAD4 concentration and its polymorphisms in SAKI development. Thus, the aim of the current study was to evaluate PAD 4 concentration and PADI4 polymorphisms as predictors of SAKI development, and among these patients, need for renal replacement therapy (RRT) and mortality.

\section{Methods}

\section{Study population}

This prospective observational clinical study, was a subanalysis of a previous study of our group that analyzed oxidative stress markers as early predictors of septic shock mortality and SAKI development [18]. We included patients admitted to the Intensive Care Unit (ICU) of our institution with the diagnosis of septic shock, from May 2014 to June 2015.

We included in the study all individuals with the diagnosis of septic shock at ICU admission, over 18 years of age, of both sexes and requiring vasopressor support for at least 24 hours. The diagnosis of septic shock and acute kidney injury were made by the medical team according to standard guidelines $[19,20]$. Baseline creatinine was characterized as the lowest creatinine value in the past 6 months before AKI or, the lowest value achieved during hospitalization in the absence of dialysis. [21,22] CKD was defined according to CKD Epidemiology Collaboration equation (CKD-EPI) [23].

Exclusion criteria were delayed diagnosis of septic shock (time greater than $24 \mathrm{~h}$ ), the presence of other types of shock, noradrenaline dose $>2.0 \mu \mathrm{g} / \mathrm{kg} / \mathrm{min}$, inability to provide consent, pregnancy, confirmed brain death and the need for palliative care. Moreover, not include patients with presence of AKI at ICU admission, stage 4 or 5 CKD (creatinine clearance less than $30 \mathrm{~mL} / \mathrm{min} / 1.73 \mathrm{~m}^{2}$ ).

Demographic, clinical and laboratory data were collected upon ICU admission. Blood samples were taken within the first 24 hours of the patient's admission to determine serum PAD4 concentration and its 
polymorphism PADI4 (rs11203367) and (rs874881). The development of SAKI was evaluated and among the patients in whom SAKI developed, mortality and need for RRT were also evaluated.

\section{Laboratory Analysis}

Laboratory tests such as CRP, albumin, lactate, urea and creatinine were measured with dry chemistry method. The hemograms were performed with a Coulter STKS hematological auto analyzer.

\section{Serum PAD4 concentration}

For determination of serum concentration of PAD4, we used enzyme-linked immunosorbent assay (CloudClone Corp, Houston, TX, USA). All instructions of the kit manufacturer were followed and the sensitivity value adopted was $0.137 \mathrm{ng} / \mathrm{mL}$.

\section{PADI4 gene polymorphism}

From whole blood samples, DNA was isolated, its integrity was confirmed on $1 \%$ agarose gel and concentration obtained by Nanodrop 8000 spectrophotometer (Thermo Scientific, Waltham, MA, USA). In addition, TaqMan Open Array (Applied Biosystem, Foster City, CA, USA) was used according to the manufacturer's instructions for the genotyping of the polymorphisms of the PAD4 gene rs 11203367 and rs87481[24].

\section{Statistical Analysis}

Data are expressed as the mean \pm standard deviation, the median (including the lower and upper quartiles), or percentage. Statistical comparisons between two groups for continuous variables were performed using the $t$-test for parameters with a normal distribution. If data were not normally distributed, comparisons between two groups were performed using the Mann-Whitney Utest. Fisher test or the chisquare test was used for all categorical data. Spearman correlation was performed to analyze the association between continuous variables. Hardy-Weinberg equilibrium was determined using chi-square test. Data analysis was performed using SigmaPlot software for Windows v12.0 (Systat Software Inc., San Jose, CA, USA). Values of $P<0.05$ were considered statistically significant.

\section{Results}

During the study, 150 consecutive patients were admitted to the ICU with the diagnosis of septic shock. However, 27 patients were excluded due to presence of AKI at ICU admission; 12 because of a delay in septic shock diagnosis; 4 because of the presence of advanced chronic kidney disease; 3 because PAD 4 concentration below assay sensitivity; and 5 had technical problems with polymorphism analysis. Thus, we evaluated 99 patients.

The mean age was $63.6 \pm 14.4$ years, $56 \%$ were male, and the median length of ICU stay was 9 (4 to 17) days. The mortality rate during the ICU stay was $69.9 \%$. Median PAD4 serum concentration was 4.4 (2.5 
to 6.2$) \mathrm{ng} / \mathrm{mL}$. Among these patients with septic shock, SAKI developed in $51.5 \%$ during the ICU stay; of these, $21.5 \%$ required RRT and $80 \%$ died.

The genotype frequencies for the rs 11203367 polymorphism were $49.59 \%$ for CT, $35.4 \%$ for CC and $15.1 \%$ for TT; for the rs 874881 polymorphism, they were $51.5 \%$ for $\mathrm{CG}, 16.2 \%$ for GG and $32.3 \%$ for CC. These frequencies are consistent with those expected under the Hardy-Weinberg equilibrium.

The demographic and laboratory data are presented in Tables 1 and 2, respectively.

Table 1. Demographic and PADI4 polymorphisms data of 99 patients with septic shock

\begin{tabular}{lccc}
\hline Variables & \multicolumn{2}{c}{ SAKI development } & $P$ \\
\cline { 2 - 3 } & Yes (n=51) & No (n = 48) & \\
\hline Age (years) & $67(59-75)$ & $64(51-71)$ & 0.093 \\
Male, n (\%) & $27(53)$ & $28(58)$ & 0.736 \\
APACHE II score & $18.8 \pm 6.0$ & $16.5 \pm 6.8$ & 0.070 \\
SOFA score & $10.4 \pm 2.7$ & $8.8 \pm 2.3$ & 0.002 \\
Sepsis focus, n (\%) & $35(69)$ & $23(48)$ & 0.203 \\
Respiratory & $11(22)$ & $12(25)$ & \\
Abdominal & $1(2)$ & $3(6)$ & \\
Urinary & $4(7)$ & $10(21)$ & \\
Outros & $41(80)$ & $28(58)$ & 0.003 \\
Mortality ICU, n (\%) & & & 0.910 \\
PADI4 (rs11203367), n (\%) & $26(51)$ & $23(48)$ & \\
CT & $17(33)$ & $18(37.5)$ & \\
CC & $8(16)$ & $7(14.5)$ & \\
TT & & & 0.769 \\
PADI4 (rs874881), n (\%) & $28(55)$ & $23(48)$ & \\
CG & $8(16)$ & $8(17)$ & \\
GG & $15(29)$ & $17(35)$ & \\
CC & & & \\
\hline
\end{tabular}

APACHE II = Acute Physiology and Chronic Health Evaluation, ICU = intensive care unit, PADI4 = polymorphisms peptidylarginine deiminase 4 , SOFA = Sequential Organ Failure Assessment. Data are expressed as the mean \pm SD. median (including the lower and upper quartiles) or percentage.

Table 2. Laboratory data of 99 patients with septic shock 


\begin{tabular}{lccc}
\hline Variable & \multicolumn{2}{c}{ SAKI development } & $P$ \\
\cline { 2 - 3 } & \multicolumn{1}{c}{ Yes $(\mathrm{n}=51)$} & No $(\mathrm{n}=48)$ & \\
\hline PAD4 $(\mathrm{ng} / \mathrm{mL})$ & $4.6(3.1-6.7)$ & $3.8(1.9-6.2)$ & 0.116 \\
Lactate $(\mathrm{mmol} / \mathrm{L})$ & $2.3(1.4-3.5)$ & $2.3(1.2-3.8)$ & 0.890 \\
Hemoglobin $(\mathrm{g} / \mathrm{dL})$ & $10.7 \pm 2.0$ & $11.4 \pm 2.1$ & 0.072 \\
Hematocrit $(\%)$ & $32.2 \pm 6.1$ & $34.0 \pm 6.1$ & 0.155 \\
Leucocytes $\left(10^{3} / \mathrm{mm}^{3}\right)$ & $17.0(13.2-22.4)$ & $16.5(11.0-24.6)$ & 0.872 \\
CRP $(\mathrm{mg} / \mathrm{dL})$ & $36.5(28.0-44.1)$ & $30.5(8.2-35.5)$ & 0.030 \\
Albumin, $(\mathrm{g} / \mathrm{dL})$ & $2.3(2.0-2.5)$ & $2.1(1.8-2.6)$ & 0.427 \\
Urea, $(\mathrm{mg} / \mathrm{dL})$ & $101(68-160)$ & $53(33-85)$ & $<0.001$ \\
Creatinine, $(\mathrm{mg} / \mathrm{dL})$ & $2.0(1.6-2.6)$ & $0.8(0.5-1.1)$ & $<0.001$ \\
\hline
\end{tabular}

$\mathrm{CRP}=\mathrm{C}$-reactive protein, PAD4 = peptidylarginine deiminase 4; SAKI = sepsis-induced acute kidney injury. Data are expressed as mean \pm standard deviation or median (including the lower and upper quartiles).

Patients with SAKI had higher Sequential Organ Failure Assessment (SOFA) score, higher serum concentration of C-reactive protein (CRP), urea, and creatinine, and higher mortality rate. There were no differences between PAD4 concentrations and its polymorphisms rs11203367 and rs87481 in patients in whom SAKI developed or not. Nevertheless, it is relevant to note that the serum concentration of PAD4 had a positive correlation with plasma urea $(r=0.269 ; p=0.007)$ and creatinine concentration $(r=0.284$; $p=0.004)$.

Patients with SAKI who died in the ICU had higher Acute Physiology and Chronic Health Evaluation (APACHE II) and SOFA scores. However, there were no differences between PAD4 concentrations and its polymorphisms rs11203367 and rs874881 in patients who died during the ICU stay (Table 3).

Table 3. Demographic, PAD 4 serum concentration and PADI4 polymorphisms data of 51 patients with SAKI 


\begin{tabular}{lccc}
\hline Variables & \multicolumn{2}{c}{ Mortality ICU } & $P$ \\
\cline { 2 - 3 } & Yes $(\mathrm{n}=41)$ & No $(\mathrm{n}=10)$ & \\
\hline Age (years) & $66.5 \pm 12.8$ & $64.5 \pm 11.4$ & 0.624 \\
Male, n (\%) & $21(51)$ & $6(60)$ & 0.731 \\
APACHE II score & $19.7 \pm 6.1$ & $15.4 \pm 4.3$ & 0.043 \\
SOFA score & $10.8 \pm 2.7$ & $8.6 \pm 1.7$ & 0.020 \\
Sepsis focus, n (\%) & $29(71)$ & $6(60)$ & \\
Respiratory & $7(17)$ & $4(40)$ & \\
Abdominal & $1(2)$ & 0.507 \\
Urinary & $4(10)$ & $(0)$ & \\
Others & $4.7(3.1-6.8)$ & $3.9(2.4-7.2)$ & 0.678 \\
PAD 4, (ng/mL) & & & 0.780 \\
PADI4 (rs11203367), n (\%) & $20(49)$ & $6(60)$ & \\
CT & $14(34)$ & $3(30)$ & \\
CC & $7(17)$ & $1(10)$ & \\
TT & & & \\
PADI4 (rs874881), n (\%) & $21(51)$ & $7(70)$ & \\
CG & $7(17)$ & $1(10)$ & \\
GG & $13(32)$ & $2(20)$ & \\
CC & & & \\
\hline
\end{tabular}

APACHE II = Acute Physiology and Chronic Health Evaluation, PAD4 = peptidylarginine deiminase 4, PADI4 = polymorphisms peptidylarginine deiminase 4, SOFA = Sequential Organ Failure Assessment. Data are expressed as the mean \pm standard deviation. median (including the lower and upper quartiles) or percentage.

Patients with SAKI who needed RRT had a higher SOFA score, but the same association was not observed with PAD4 concentration and PADI4 polymorphisms (Table 4).

Table 4. Demographic, PAD4 serum concentration and PADI4 polymorphisms data of 51 patients with SAKI 


\begin{tabular}{lccc}
\hline Variables & \multicolumn{2}{c}{ RRT ICU } & $P$ \\
\cline { 2 - 3 } & Yes $(\mathrm{n}=11)$ & No $(\mathrm{n}=40)$ & \\
\hline Age (years) & $63.2 \pm 14.1$ & $67.1 \pm 12.1$ & 0.365 \\
Male, n (\%) & $4(36)$ & $23(58)$ & 0.367 \\
APACHE II score & $21.8 \pm 6.4$ & $18.0 \pm 5.7$ & 0.061 \\
SOFA score & $12.0(12.0-15.0)$ & $9.5(8.0-11.0)$ & 0.003 \\
PAD4 (ng/mL) & $4.6(3.1-9.3)$ & $4.5(2.8-6.1)$ & 0.384 \\
PADI4 (rs11203367), n (\%) & & & 0.102 \\
CT & $4(36.5)$ & $22(55)$ & \\
CC & $4(36.5)$ & $14(35)$ & \\
TT & $3(27)$ & $4(10)$ & 0.094 \\
PADI4 (rs874881), n (\%) & $4(36.5)$ & $24(60)$ & \\
CG & $4(36.5)$ & $4(10)$ & \\
GG & $3(27)$ & $12(30)$ & \\
CC & & &
\end{tabular}

APACHE II = Acute Physiology and Chronic Health Evaluation; ICU = intensive care unit, SOFA = Sequential Organ Failure Assessment, PAD 4 = peptidylarginine deiminase 4; PADI4: polymorphisms peptidylarginine deiminase 4, RRT, renal replacement therapy. Data are expressed as the mean \pm standard deviation, median (including the lower and upper quartiles) or percentage.

\section{Discussion}

The aim of the current study was to evaluate PAD4 concentration and PADI4 polymorphisms as predictors of SAKI development, and among these patients, the need for RRT and mortality. This study showed that PAD4 concentration and its polymorphisms were not associated with SAKI development or with the other outcomes. However, PAD4 concentrations were associated with creatinine and urea levels in patients with septic shock.

SAKI is a frequent complication in patients with sepsis and is associated with adverse outcomes including increased length of hospital stay, development of CKD, and increased risk of death [25,26]. Despite efforts to obtain early diagnosis and treatment of septic shock, mortality rates remain high [18]. In our study, $69.9 \%$ of patients with septic shock died. In addition, in patients in whom SAKI developed, the mortality was even greater, representing $80 \%$ of the cases. Although very high, our rates are in agreement with data found for Latin America $[18,27]$.

The pathophysiology of AKI induced by sepsis is multifactorial and complex, and the development of early and effective therapeutic targets is fundamental. In this scenario, PAD4 could play an important role. Currently, it is believed that the process of renal ischemia-reperfusion contributes to the increase of PAD4 expression. Consequently, there would be a stimulus for the release of proinflammatory cytokines, renal neutrophils infiltration, and histone citrullination, which together would corroborate the formation of NETs. The disconnected formation of NETs seems to stimulate the formation of thrombi, contributing to renal tubular cell necrosis. In addition, patients with septic shock have a high proinflammatory response, reduced immune capacity, and tissue hypoperfusion, which together with increased NET formation create 
a vicious and extremely lethal cycle [11]. In this context, experimental studies suggest that blocking the action of PAD4 using drugs or genetically have a protective effect against ischemic AKI [28].

In general, studies available in the literature only address ischemic AKI in experimental models, and this is the first clinical study to evaluate the influence of PAD4 and its polymorphisms in SAKI. In this study, AKI developed in half of the patients during hospitalization, which contributes to septic shock severity and survival reduction. Despite this, we did not observe a difference in the concentration of PAD4 between the individuals in whom AKI developed and those who died. This response is different from what we found in our previous study, with the same dataset, in which PAD4 concentration was associated with increased mortality in patients with septic shock [15]. This different performance of PAD4 as a biomarker suggests that PAD4 concentration is more important in sepsis than in AKI pathophysiology. Importantly, despite the lack of significant results, we did not rule out the influence on PAD4 in the development and worse evolution of AKI, because its higher concentration was associated with increased levels of creatinine and urea in patients with septic shock.

It is already known that functional polymorphisms could influence enzyme activity and patient evolution. In this work, we evaluated only two PADI4 polymorphisms (rs 11203367, rs 87481) and none of them had an association with the development of SAKI, or need for RRT and mortality. In addition, our previous study also showed in patients with septic shock that other PADI4 polymorphisms (rs 11203366, rs 2240340, rs 1748033) were not associated with mortality [15]. We also could not exclude the participation other PADI4 polymorphisms in SAKI development and outcomes.

Some limitations of this study should be considered. We only included patients from a single center and our sample size was relatively small. Regarding diagnosis of AKI, it was based on serum creatinine values and not on urine output. Moreover, the only marker of inflammation that we measured was CRP and we did not evaluate the formation of NETs. Despite these limitations, we strongly believe that our data contribute to a better knowledge of inflammatory pathways in patients with SAKI.

\section{Conclusion}

In conclusion, PAD4 concentrations were associated with creatinine and urea levels in patients with septic shock. However, PAD4 concentration and its polymorphisms were not associated with SAKI development, need for RRT and mortality in these patients.

\section{List Of Abbreviations}

APACHE II - Acute Physiology and Chronic Health Evaluation; CKD - Chronic kidney disease; CRP - Creactive protein; ICU - Intensive Care Unit; NETs - Neutrophil extracellular traps; PAD - Peptidyl arginine deiminase; PAD4 - Peptidyl arginine deiminase 4; RNA - Ribonucleic acid; RRT - Renal replacement therapy; SOFA - Sequential Organ Failure Assessment. 


\section{Declarations}

\section{Acknowledgments}

None.

\section{Funding}

This study was funded by the State of São Paulo Research Foundation (FAPESP - 2014/17262-0; 2014/07988-4) and CAPES ("Coordenação de Aperfeiçoamento de Pessoal de Nível Superior").

The funding had no role in the design of the study, collection, analysis, and interpretation of data and in writing the manuscript.

\section{Availability of data and materials}

The datasets used and/or analyzed during the current study are available from the corresponding author on reasonable request.

\section{Competing interests}

The authors declare that they have no competing interests.

\section{Consent for publication}

Not applicable.

\section{Ethics approval and consent to participate}

The research was initiated only after approval by the Research Ethics Committee of our institution under protocol number 30457414.7.0000.5411. The work described was carried out in accordance with The Code of Ethics of the World Medical Association (Declaration of Helsinki). Written informed consent was obtained from all patients or their legally authorized surrogate, prior to their inclusion in the study.

\section{Author's contributions}

PSA, AGP, BFB, ALG, RVSB, DP, ALB: acquisition of subjects and data, analysis and interpretation of data, revising the article critically; NAC, SARP, MFM, LAMZ: study design, analysis and interpretation of data, statistical analysis and drafting the manuscript. All authors contributed important intellectual content during manuscript drafting or revision and accepts accountability for the overall work by ensuring that questions pertaining to the accuracy or integrity of any portion of the work are appropriately investigated and resolved.

\section{References}


1. Clark E, Bagshaw SM. Long-term risk of sepsis among survivors of acute kidney injury. Crit Care. 2014;18(1):1-3.

2. Jo SK, Rosner MH, Okusa MD. Pharmacologic treatment of acute kidney injury: why drugs haven't worked and what is on the horizon. Clin J Am Soc Nephrol. 2017;2(2):356-365.

3. Suh SH, Kim CS, Choi JS, Bae EH, Ma SK, Kim SW. Acute kidney injury in patients with sepsis and septic shock: risk factors and clinical outcomes. Yonsei Med J. 2013;54(4): 965-72.

4. Ramesh G, Reeves WB. Inflammatory cytokines in acute renal failure. Kidney Int Suppl. 2004; (91)66:S56-S61.

5. Frangogiannis NG. Chemokines in ischemia and reperfusion. Thromb Haemost. 2007; 97(5):738747.

6. Heinzelmann M, Mercer-Jones MA, Passmore JC. Neutrophils and renal failure. Am J Kidney Dis. 1999;34(2):384-399.

7. Bak Z, Gyorgy B, Géher P et al. Citrullination under physiological and pathological conditions. Joint Bone Spine. 2012;79(5):431-436.

8. Nguyen H, James EA. Immune recognition of citrullinated epitopes. Immunology. 2016;149(2):131138.

9. Jang B, Ishigami A, Maruyama N, Carp RI, Kim YS, Choi EK. Peptidylarginine deiminase and protein citrullination in prion diseases: strong evidence of neurodegeneration. Prion. 2013;7(1):42-46.

10. Anzilotti C, Pratesi F, Tommasi C, Migliorini P. Peptidylarginine deiminase 4 and citrullination in health and disease. Autoimmun Rev. 2010;9(3):158-160.

11. Martinod K, Demers M, Fuchs TA, Wong SL, Brill A, Gallant M et al. Neutrophil histone modification by peptidylarginine deiminase 4 is critical for deep vein thrombosis in mice. Proc Natl Acad Sci. 2013;110(21):8674 -8679.

12. van Beers JJ, Zendman AJ, Raijmakers R, Stammen-Vogelzangs J, Pruijn GJ. Peptidylarginine deiminase expression and activity in PAD2 knock-out and PAD4-low mice. Biochimie. 2013;95(2):299 $-308$.

13. Vossenaar ER, Zendman AJ, van Venrooij WJ, Pruijn GJ. PAD, a growing family of citrullinating enzymes: genes, features and involvement in disease. Bioessays. 2003;25(11): $1106-1118$.

14. Ham A, Rabadi M, Kim M, Brown KM, Ma Z, D'Agati V et al. Peptidyl arginine deiminase-4 activation exacerbates kidney ischemia-reperfusion injury. Am J Physiol Renal Physiol. 2014;307(9):F10521062.

15. Costa NA, Gut AL, Azevedo PS, Polegato BF, Magalhães ES, Ishikawa LLW et al. Peptidylarginine deiminase 4 concentration, but not PADI4 polymorphisms, is associated with ICU mortality in septic shock patients. J Cell Mol Med. 2018;22(10):4732-4737.

16. Kobayashi T, Ito S, Kobayashi D, Shimada A, Narita I, Murasawa A et al. Serum immunoglobulin G levels to porphyromonas gingivalis peptidylarginine deiminase affect clinical response to biological disease-modifying antirheumatic drug in rheumatoid arthritis. Plos One. 2016;11(4):e0154182. 
17. Lee YH, Bae SC. Association between susceptibility to rheumatoid arthritis and PADI4 polymorphisms: a meta-analysis. Clin Rheumatol. 2016;35(4):961-971.

18. Costa NA, Gut AL, Azevedo PS, Tanni SE, Cunha NB, Magalhães ES et al. Erythrocyte superoxide dismutase as a biomarker of septic acute kidney injury. Ann Intensive Care. 2016;6(1):95.

19. Singer M, Deutschman CS, Seymour CW, Shankar-Hari M, Annane D, Bauer M et al. The Third International Consensus Definitions for Sepsis and Septic Shock (Sepsis-3). JAMA. 2016;315(8):801810.

20. KDIGO AKI Work Group: KDIGO clinical practice guideline for acute kidney injury. Kidney Int Suppl. 2012;2(1):1-138.

21. Gammelager H, Christiansen CF, Johansen MB, Tønnesen E, Jespersen B, Sørensen HT. Five-year risk of end-stage renal disease among intensive care patients surviving dialysis-requiring acute kidney injury: a nationwide cohort study. Crit Care. 2013;17(4): R145.

22. Siew ED, Matheny ME, Ikizler TA, Lewis JB, Miller RA, Waitman LR et al. Commonly used surrogates for baseline renal function affect the classification and prognosis of acute kidney injury. Kidney Int. 2010;77(6):536-542.

23. Levey AS, Stevens LA, Schmid CH, Zhang YL, Castro AF, Feldman HI et al. A new equation to estimate glomerular filtration rate. Ann Intern Med. 2009;150(9):604-612.

24. Norde MM, Oki E, de Castro IA, Souza JMP, Damasceno NRT, Fisberg RM et al. Influence of adiponectin gene variants and plasma fatty acids on systemic inflammation state association-A cross-sectional population-based study, São Paulo, Brazil. Mol Nutr Food Res. 2016;60(2):278-286.

25. Chawla LS, Eggers PW, Star RA, Kimmel PL. Acute kidney injury and chronic kidney disease as interconnected syndromes. N Engl J Med. 2014;371(1):58-66.

26. Bellomo R, Kellum JA, Ronco C. Acute kidney injury. Lancet. 2012;380(9846):756-766.

27. Machado FR, Cavalcanti AB, Bozza FA, Ferreira EM, Angotti Carrara FS, Sousa JL et al. The epidemiology of sepsis in Brazilian intensive care units (the Sepsis PREvalence Assessment Database, SPREAD): an observational study. Lancet Infect Dis. 2017;17(11): 1180-1189.

28. Jing L, Zheng D. Neutrophil extracellular traps in ischemic AKI: new way to kill. Kidney International. 2018; 93(2):303-305. 